\title{
Predictive Tree: An Efficient Index for Predictive Queries On Road Networks
}

\author{
Abdeltawab M. Hendawi ${ }^{1,2} \quad$ Jie Bao $^{1} \quad$ Mohamed F. Mokbel $^{1} \quad{\text { Mohamed } \mathrm{Ali}^{2,3}}^{2,3}$ \\ ${ }^{1}$ Department of Computer Science and Engineering, University of Minnesota, Minneapolis, MN, USA \\ ${ }^{1}$ \{hendawi, baojie, mokbel\}ecs.umn.edu \\ ${ }^{2}$ Institute of Technology, University of Washington, Tacoma, WA, USA \\ ${ }^{2}$ \{hendawi, mhali\}euw.edu \\ ${ }^{3}$ Microsoft Corporation \\ ${ }^{3}$ maliemicrosoft.com
}

\begin{abstract}
Predictive queries on moving objects offer an important category of location-aware services based on the objects' expected future locations. A wide range of applications utilize this type of services, e.g., traffic management systems, location-based advertising, and ride sharing systems. This paper proposes a novel index structure, named Predictive tree (P-tree), for processing predictive queries against moving objects on road networks. The predictive tree: (1) provides a generic infrastructure for answering the common types of predictive queries including predictive point, range, $K N N$, and aggregate queries, (2) updates the probabilistic prediction of the object's future locations dynamically and incrementally as the object moves around on the road network, and (3) provides an extensible mechanism to customize the probability assignments of the object's expected future locations, with the help of user defined functions. The proposed index enables the evaluation of predictive queries in the absence of the objects' historical trajectories. Based solely on the connectivity of the road network graph and assuming that the object follows the shortest route to destination, the predictive tree determines the reachable nodes of a moving object within a specified time window $\mathcal{T}$ in the future. The predictive tree prunes the space around each moving object in order to reduce computation, and increase system efficiency. Tunable threshold parameters control the behavior of the predictive trees by trading the maximum prediction time and the details of the reported results on one side for the computation and memory overheads on the other side. The predictive tree is integrated in the context of the $i$ Road system in two different query processing modes, namely, the precomputed query result mode, and the on-demand query result mode. Extensive experimental results based on large scale real and synthetic datasets confirm that the predictive tree achieves better accuracy compared to the existing related work, and scales up to support a large number of moving objects and heavy predictive query workloads.
\end{abstract}

\section{INTRODUCTION}

The availability of hundreds of millions of smart phones [6] in users' hands during their movements in daily lives fired the explosion of a vast number of location aware services [5], [11], [20], [29]. Predictive queries [10], [12], [13] offer a fundamental type of location-based services based on users' future locations. Common types of predictive spatial queries include predictive range query, e.g., "find all hotels that are

This work is partially supported by the National Science Foundation, USA, under Grants IIS-0952977 and IIS-1218168. located within two miles from a user's anticipated location after 30 minutes", predictive KNN query, e.g., "find the three taxis that are closest to a user's location within the next 10 minutes", and predictive aggregate query, e.g., "find the number of cars expected to be around the stadium during the next 20 minutes".

In fact, Predictive queries are beneficial in various types of real applications such as (1) traffic management, to predict areas with high traffic in the next half hour, so appropriate decisions are taken before congestion appears, (2) locationaware advertising, to distribute coupons and sales promotions to customers more likely to show up around a certain store during the sale time in the next hour, (3) routing services, to take into consideration the predicted traffic on each road segment to find the shortest path of a user's trip starting after 15 minutes from the present time, (4) ride sharing systems, to match the drivers that will pass by a rider's location within few minutes, and (5) store finders, to recommend the closest restaurants to a user's predicted destination in 15 minutes.

In this paper, we address the problem of how to process predictive queries for moving objects on road networks efficiently. To this end, we introduce a novel index structure, named the Predictive tree ( $P$-tree), proposed to precompute the predicted moving objects around each node in the underlying road network graph over time. The predictive tree is best described as generic and extensible, from a functionality perspective, dynamic and tunable from a performance perspective.

\section{A. Challenges}

Existing studies on predictive query processing have gone a long way in advancing predictive location-based services. However, existing techniques suffer from both functional limitations and performance deficiencies. From a functional perspective, they suffer from one or more of the following limitations: (1) They consider an Euclidean space [9], [28], [25], [30] where objects can move freely in a two dimensional space. Yet, practical predictive location-based services target moving objects on road networks as described by the motivating applications earlier in this section. (2) Many techniques utilize prediction models that must be trained using a massive 
a mount of objects' historical trajectories in order to produce accurate predictions [2], [9], [13], [15], [24], [28]. However, practical scenarios and industrial experience reveal that such historical data is not easily obtainable for many reasons, either due to users' privacy and data confidentiality on one side or due to the unavailability of historical data in rural areas on the other side. (3) Most of the previous solutions were designed to support a specific query type only, e.g., [13], [25], [30] support predictive range query, [3], [22], [30] support predictive $K \mathrm{NN}$ query, and [9], [24] support predictive aggregate query.

\section{B. Approach}

Before summarizing the contributions of the proposed predictive tree index, we briefly highlight the basic idea of the index in order to build the proper context. Once an object starts a trip, we construct a predictive tree for this object such that the object's start node in the road network graph becomes the root of the tree. The predictive tree consists of the nodes reachable within a certain time frame $\mathcal{T}$ from the object's start location. More specifically, we assume that moving objects follow shortest paths during their travel from source to destination [16], [18]. Hence, we organize the nodes inside the predictive tree according to the shortest path from the object's start node, which is marked as the root of the tree. Accordingly, each branch from the root node to any node in the tree represents the shortest route from the root to this node.

Then, our prediction is based on a probability assignment model that assigns a probability value to each node inside the object's predictive tree. In general, the probability assignment is made according to the node's position in the tree, the travel time between the object and this node and the number of the sibling nodes. In practice, the probability assignment process is tricky and varies from one application to another.

At each node in the given road network that is indexed in $R$-tree, we keep track of a list of objects predicted to appear in this node, along with their probabilities, and travel time cost from the objects' current locations to this node. This list represents a raw precomputed answer that can be customized according to the type of the received query (i.e., point, range or $k \mathrm{NN}$ predictive query) at query processing time. When an object moves from its current node to a different node, we incrementally update the predictive tree by pruning all nodes in the tree that are no longer accessible through a shortest route from the object's new location. Mostly, this pruning shrinks the number of possible destinations, yet, increases the focus of the prediction. Consequently, the precomputed answer at each node in the object predictive tree is updated to accommodate the effect of the object's movements. This update is reflected to the original nodes in the road network. When a predictive query is received, we fetch the up-to-date answer from the node of interest and compile it according to the query type.

To adjust the behavior of the predictive tree and, hence, control the overall predictive query processing performance, we leverage two tunable parameters, a maximum time $\mathcal{T}$ and a probability threshold $\mathcal{P}$. These parameters compromise between the maximum prediction time a predictive tree can support and the details in the reported query results on one side, and system resources overheads, i.e., CPU and memory, on the other side.

The proposed predictive tree is implemented within the $i$ Road framework. The iRoad offers two query processing modes of leveraging the predictive tree to control the interaction between its components: (1) the precomputed query result mode, in which the predicted results are computed and materialized in advance; and (2) the on-demand query result mode which is a lazy approach that postpones all computation till a query is received.

\section{Contributions}

In general, the contributions of this paper can be summarized as follows:

- We propose a novel data structure named Predictive tree ( $P$-tree) that supports predictive queries against moving objects on road networks.

- We introduce a probability model that computes the likelihood of a node in the road network being a destination to a moving object. The probability model is introduced to the predictive tree as a user defined function and is handled as a black box by the index construction and maintenance algorithms.

- We introduce two tunable parameters $\mathcal{T}$ and $\mathcal{P}$ that are experimentally proved to be efficient tools to control the predictive tree index, the system performance, the prediction time, and the results details as well.

- We provide an incremental approach to update the predictive tree as objects move around. Hence, we utilize the existing index structure and incur minimal cost in response to the movement of the object.

- We propose the iRoad framework that leverages the introduced predictive tree to support a wide variety of predictive queries including predictive point, range, and $K \mathrm{NN}$ queries.

- we provide an experimental evidence based on real and synthetic data that our introduced index structure is efficient in terms of query processing, scalable in terms of supporting large number of moving objects and heavy query workloads, and achieves a high-quality prediction without the need to reveal objects' historical data.

The remainder of this paper is organized as follows. Section II sets the preliminaries and defines our problem. Section III presents the iRoad system. Section IV describes the the predictive tree and its associated construction, maintenance and querying algorithms. Experimental results are presented in Section V. The study of related work is given in Section VI. Finally, Section VII concludes the paper.

\section{Preliminaries}

In this section, we formalize the basic predictive query we address in this paper. Then, we define different types of predictive queries that the predictive tree can support within the iRoad framework. After that, we explain the intuition of the leveraged prediction model. 


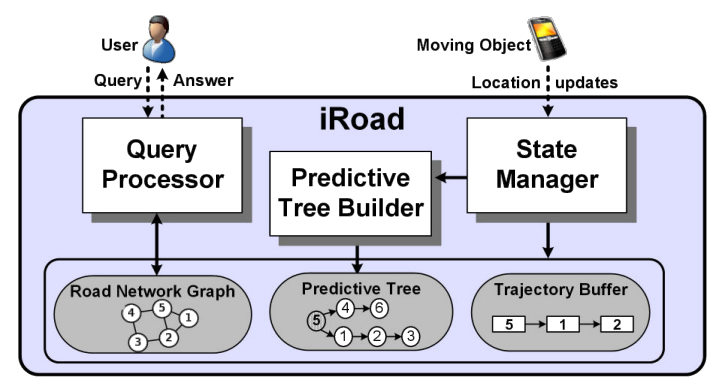

Fig. 1. iRoad System Architecture

\section{A. Basic Query}

In this paper, we focus on addressing the predictive point query as our basic query on the road network. In this query, we want to find out the moving objects with their corresponding probabilities that are expected to be around a specified query node in the road network within a future time period. The example of such query could be like, "Find out all the cars that may pass by my location in the next 10 mins". The predictive point query we address in this paper can be formalized as: "Given (1) a set of moving objects $\mathcal{O}$, (2) a road network graph $G(N, E, W)$, where $N$ is the set of nodes, $E$ is the set of edges, and $W$ is the edge weights, i.e., travel times, and (3) a predictive point query $Q(n, t)$, where $n \in N$, and $t$ is a future time period, we aim to find the set of objects $R$ $\in \mathcal{O}$ expected to show up around the node $n$ within the future time $t$. The returned result should identify the objects along with their probabilities to show up at the node of interest. For example, within the next 30 mins, object $o_{1}$ is expected to be at node $n_{3}$ with probability $0.8, R\left(Q\left(n_{3}, 30\right)\right)=\left\{\left\langle o_{1}, 0.8\right\rangle\right\}$.

\section{B. Extensions}

We consider the aforementioned predictive point query as a building block upon which our framework can be extended to support other types of predictive queries including: (i) Predictive range query, where a user defines a query region that might contain more than one node and asks for the list of objects expected to be inside the boundaries of that region within a specified future time, (ii) Predictive $K N N$ query to find out the most likely $K$ objects expected to be around the node of interest within a certain time period, and (iii) Predictive aggregate query to return the number of objects predicted to be within a given location in the next specified time duration.

\section{Prediction Model}

Our prediction model employed by the introduced predictive tree index structure is based on two corner stones. (1) The assumption that objects follow the shortest paths in their routing trips. The intuition behind this assumption is based on the fact that in most cases, the moving objects on road networks, e.g., vehicles, travel through shortest routes to their destinations [16], [18]. In fact, this assumption is aligned with the observation in [4] that moving objects do not use random paths when traveling through the road network, rather they follow optimized ones, e.g., fastest route. As a result, this

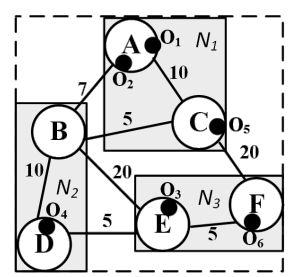

(a) Network \& Objects
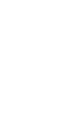

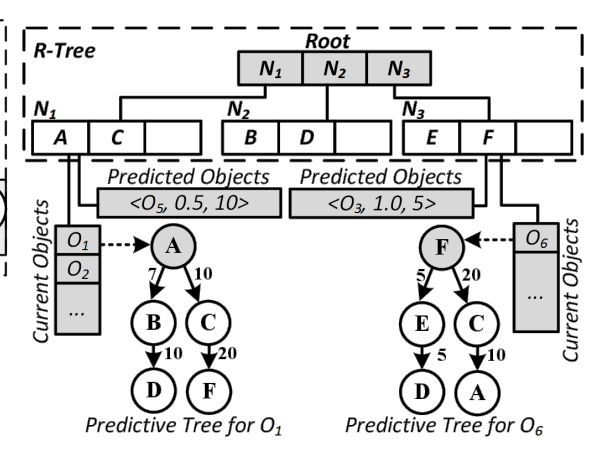

(b) Predictive Trees Integrated With R-Tree
Fig. 2. Example Of The Proposed Index Structure

model prevents the looping case that appears in the traditional turn-by-turn probability model and assigns a probability value for the moving object to turn when facing an intersection [13].

(2)The probability assignment model that assigns a probability value to each node inside the object's predictive tree. In fact, the probability assignment is affected by the node's position with respect to the root of the tree, the travel time cost between the object in its current location to this node, and the number of the sibling nodes. In general, our predictive tree is designed to work with different probability assignment models. For example, a possible probability model can give higher values to nodes in business areas, e.g., down town, rather than those in the suburbs. In our default probability model, each node in the predictive tree has a value equal to one divided by the number of nodes accessible from the root within a certain time range.

\section{The IRoAd System}

The proposed predictive tree is implemented in the context of the iRoad System. More precisely, the predictive tree and its construction, maintenance and querying algorithms form the core of the iRoad System. The iRoad System is a scalable framework for predictive query processing and analysis on road networks. The architecture of the iRoad system consists of three main modules, namely, the state manager, the predictive tree builder and the query processor, Figure 1. In this section, we present an overview of the iRoad System and give a brief description of its key components. Moreover, we focus on the interaction and workflow between these components under both the precomputed query result mode and the ondemand query result mode.

\section{A. State Manager}

The state manager is a user facing module that receives a stream of location updates from the moving objects being monitored by the system. The state manager maintains the following data structures. (1) An R-tree [7] that is generated on the underlying road network graph. It differs from the conventional $R$-tree in that at each leaf node, i.e., a node in the road network, in addition to storing the corresponding MBR, it also keeps track of two lists: (a) current objects that records the pointers to the objects around this node, and (b) 
predicted objects that maintains the predicted results of the objects that most likely to show up around that node. (2) A trajectory buffer that stores the most recent one or more nodes in the road network that are visited by the moving object in its ongoing trip. (3) A predictive tree such that root of a predictive tree is the current location of the moving object. Figure 2(a) gives an example of a set of objects moving on a road network, while Figure 2(b) depicts how the predictive trees are integrated within the basic data structures layout to facilitate the processing of predictive queries.

As we mentioned, the system can be running under either (1) a precomputed query result mode or (2) an on-demand query result mode. The first is the default mode inside the iRoad framework. In either modes, upon the receipt of a location update of a moving object, the $R$-tree is consulted and the new location is mapped to its closest node $N_{\text {new }}$ in the road network. If the new node $N_{\text {new }}$ is the same as the object's old node $N_{\text {old }}$, the object movement is not significant enough to change the system's state and no further action is taken. Otherwise, the object has moved to a different node and an evaluation of the impact of the object's movement is triggered in the system. We differentiate between the precomputed and the on-demand query result modes as follows.

Precomputed query result mode: In this mode, the predictive tree builder is invoked immediately once the moving object changes its current node and, consequently, the predictive tree is either constructed from scratch or updated in response to the object's movement. Remember that the predictive tree is constructed from scratch if the incoming location update belongs to a new object that is being examined by the system for the first time. Also, the predictive tree is constructed from scratch if $N_{\text {new }}$ is not a child of the root of the object's inhand predictive tree. As will be described in Section IV, this case happens if the object decided not to follow the shortest path, e.g., made a u-turn or started a new trip. Otherwise, the tree is incrementally maintained. Note that, in this mode, the trajectory buffer data structure boils down to one single node (i.e., the current node) of the moving object because of the eagerness to update the predictive tree with the receipt of every location update. Hence, the past trajectory is entirely factored in the predictive tree.

On-demand query result mode: In this mode, the trajectory buffer stores all nodes the moving object passed by since the start of its current trip. Initially, We do not perform any computation until a query is received. Then, we identify the vicinity nodes within the time range determined by the query. Those nodes might contribute in the predicted results. For each object in these nodes, we construct its predictive tree and run a series of updates according to the list of passed nodes in its trajectory buffer, Figure 3. For example, in this figure, nodes $A, G$, and $E$ are within the time range specified in the query at node $B$. Then, we construct the predictive tree for each object, $O_{1}, O_{2}, O_{3}$, in those nodes and update them according the passed nodes by each one. Obviously, $O_{1}, O_{3}$ will contribute in the predicted objects at node $B$, while $O_{2}$ will not contribute as node $B$ is no longer a possible destination

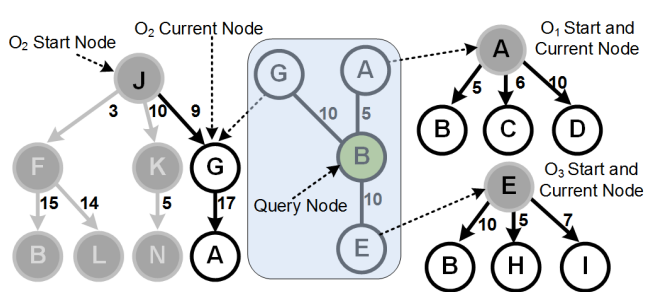

Fig. 3. On-Demand Approach

for $\mathrm{O}_{2}$ based on its trajectory buffer. Then, we get rid of any data structure, i.e., the predictive trees and predicted results, directly once the query processing is completed and the results are carried back to the query issuer. We ending by adding the object's current node $N_{\text {new }}$, i.e,. Node $B$ in this example, to the object's trajectory buffer.

\section{B. Predictive Tree Builder}

The predictive tree builder is the component that encompasses the predictive tree construction and maintenance algorithms. It takes as input, (1) the moving object's trajectory buffer, (2) the moving object's current predictive tree (if exists), (3) the tunable parameters $(\mathcal{T}$ and $\mathcal{P})$ that trade the prediction length and accuracy for system's resources, and (4) a user defined probability assigned function. The predictive tree builder reflects the most recent movements of the object (as recorded in the object's trajectory buffer) to the object's predictive tree. Upon the completion of a successful invocation of the tree builder, an up-to-date predictive tree rooted at the object's current location is obtained and the object's trajectory buffer is modified to accommodate the object's current node.

The predictive tree builder is invoked in two different ways. In a precomputed query result mode, the builder is invoked by the state manager upon the receipt of every location update. The state manager pushes the incoming location update of a moving object $O_{i}$ to the predictive tree builder that eagerly reflects the location update in the predictive tree of $O_{i}$. Afterwards, the tree builder updates the precomputed query results at every node in the road network that is on the shortest path route from the object $O_{i}$ 's current location.

In an on-demand query result mode, the predictive tree builder is invoked by the query processor once a query $Q$ is received. The predictive tree builder consults the road network graph and retrieves a list of nodes $N_{\text {vicinity }}$ that are within the time distance determined by the query $Q$. Then, it pulls, from the state manager, the predictive trees and the trajectory buffers of moving objects whose current nodes are in $N_{\text {vicinity }}$. In other words, lazy or selective processing of moving objects that are believed to affect the query result is carried over without taking the burden of updating the predictive tree of every single moving object in the system.

\section{Query processor}

The main goal behind predictive query processing in the iRoad system is to be generic and to provide an infrastructure for various query types. This goal is achieved by mapping a query type to a set of nodes $\left(N_{\text {vicinity }}\right)$ in the road network graph such that the query result is satisfied by predictions 


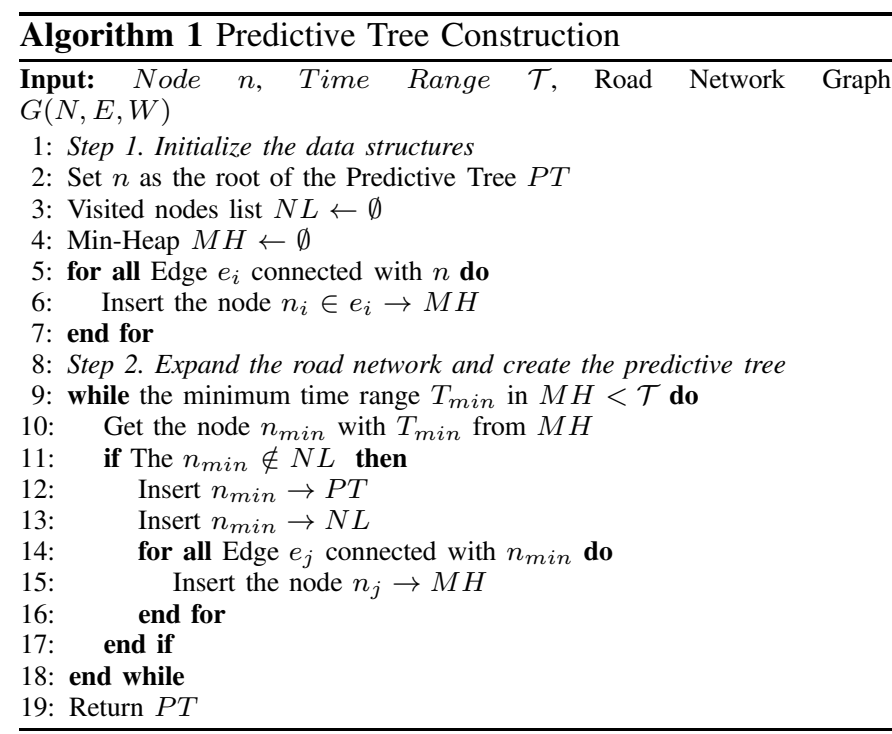

associated with these nodes. For predictive point queries as an example, the point query is answered using the information associated with the road network node that is closest to the query point. For predictive range queries, all nodes in the range are considered to compute the query result. For predictive $K \mathrm{NN}$ queries, we sort those predicted objects associated with $N_{\text {vicinity }}$ based on their probabilities. $N_{\text {vicinity }}$ is rationally expanded till $K$ objects are retrieved, if visible.

In a precomputed query result mode, generic results are prepared in advance and are held in memory. The process is triggered by an update in object's location and the precomputed results are constructed/updated for all nodes along the shortest path route of that object. Therefore, most of the work is done during the location update time. Upon the receipt of a query, the query processor fetches the precomputed results only from nodes in $N_{\text {vicinity }}$, adapts them according to the type of the received query and gives a low latency response back to the user.

In an on-demand query result mode, nothing is precomputed in advance and all computation will be performed after the receipt of the user's query. $N_{\text {vicinity }}$ is identified and the predictive tree of objects whose current node belong to $N_{\text {vicinity }}$ are constructed/upadted as described earlier in this section. Then, the results are collected and adapted to the query type in a similar way to the precomputed result approach.

\section{PREDictive TREe}

In this section, we describe the proposed predictive tree index structure that is leveraged inside the iRoad framework to process predictive queries based on the predicted destinations of the moving objects within a time period $\mathcal{T}$. We first introduce the main idea and the motivation to build the predictive tree. After that, we provide a detailed description for the two main operations in the predictive tree: 1) predictive tree construction, and 2) predictive tree maintenance.

The idea of the predictive tree is to identify all the possible destinations that a moving object could visit in a time period $\mathcal{T}$ by traveling through the shortest paths. As there may only exist one shortest path from a start node to a destination node, we can guarantee it will be a tree structure (i.e., without any loop). The intuition for constructing the predictive tree with a time boundary $\mathcal{T}$ is based on two real facts: 1 ) most of the moving objects travel through shortest path to their destinations [16], [18], and 2) majority of the real life trips are within a time period, e.g., 19 minutes [17], [18]. As a result, we only need to care about the possible destinations reachable through a shortest route from the object's start location within a bounded time period. Based on that, we build the predictive tree to hold only the accessible nodes around a moving object and assign a probability for each one of them.

The predictive trees leveraged in the iRoad system significantly improves the predictive query processing efficiency for two main reasons. 1) The possible destinations of the prediction shrinks as a result of using the time boundary $\mathcal{T}$. Yet, prediction computation is performed on few number of nodes instead of millions of nodes in the underlying road network, e.g., road network of California state in USA has about 1,965,206 nodes and 5,533,214 edges [23]. 2) Inside the predictive tree, we maintain only those nodes with probability higher than a certain probability threshold parameter $\mathcal{P}$, e.g., $10 \%$. By doing this, we cut down the computation overhead consumed for continuously maintaining the predicted results at each node in the predictive trees. Moreover, we control iRoad to focus on those nodes that more likely to be reached by a moving object. Yet, the query reported results can be more reasonable to users.

\section{A. Predictive Tree Construction}

Main idea. When a moving object starts its trip on the road network, we build a predictive tree based on its starting location to predict its possible destinations within a certain time frame $\mathcal{T}$. We propose a best-first network expansion algorithm for constructing predictive tree for time period $\mathcal{T}$, e.g., 30 minutes. We set the object's initial node as the start node, then, we visit the nodes and edges on the road network that are reachable using a shortest path from this start node [21]. The algorithm proceeds to traverse and process the edges in the road network based on the travel time cost from the start node until all the costs to the remaining edges are over $\mathcal{T}$.

Algorithm. The pseudo code for the predictive tree construction algorithm is given in Figure 1. The algorithm takes the road network $G=\{N, E, W\}$, a starting node $n$ and a time range $\mathcal{T}$ as input. The algorithm consists of two main steps:

- Initialization. We first initialize the predictive tree under construction by setting the start node $n$ as the root of the tree. We also create the visited nodes list $N L$ to store the nodes that have been processed by the algorithm so far. An empty min-heap, $M H$, is employed to order the nodes based on its distance to the root node $n$. After that, we insert the nodes that are directly connected with the 


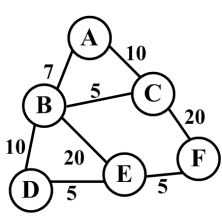

(a) Road Network

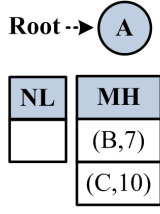

(b) Initialize Tree

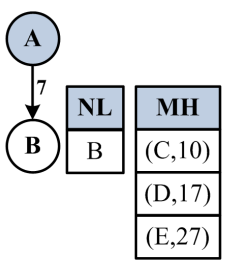

(c) Expand B

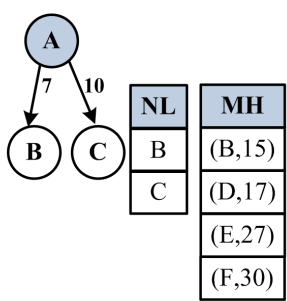

(d) Expand C

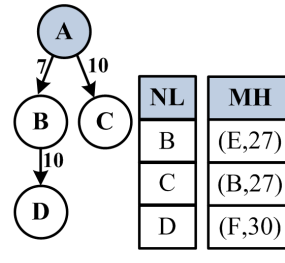

(e) Expand D

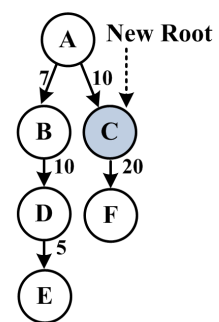

(f) Update Tree

Fig. 4. Example of Constructing And Expanding The Predictive Tree Started At Node A.

root node $n$ into the min-heap $M H$, (Lines from 2 to 7 in Algorithm 1).

- Expansion. We continuously pop the node $n_{\min }$ that is the closest to the root node from the min-heap. Then, we check if that node has been visited by our algorithm before, which means there was a shorter path from the root to this node $n_{\text {min }}$. If visited nodes list $N L$ does not contain $n_{\min }$, we insert the node $n_{\min }$ to it as well as a child to the current expanding branch of the predictive tree $P T$. After that, we insert to the min-heap $M H$ the node $n_{j}$ that is connected with the yet processed node $n_{\text {min }}$ for further expansion. The algorithm stops when the distance between the next closest node in the minheap is over the boundary $\mathcal{T}$, (Lines from 8 to 18 in Algorithm 1).

Example. Figure 4 gives an example for constructing a predictive tree for node $A$ from the given road network. For this example, we set the time period $\mathcal{T}$ to 20 minutes. Figure 4(a) gives the original road network structure, where circles represent nodes and lines between nodes represent edges and the number on each edge represents the time cost to traverse that edge. In the first iteration, we start by setting the root of the tree to node $A$. Then, we insert nodes $B$ and $C$ into the min-heap, as they are the connected ones to the root node $A$, Figure 4(b). After that, we expand the closest child to the root, $B$, where we insert $D$ and $E$ into the minheap $M H$ and put $B$ in the predictive tree and the visited nodes list $N L$ as well, Figure 4(c). In the second iteration, node $C$ is the closest node to the root with travel time cost equals 10 minutes. Yet, we insert $C$ into the predictive tree in addition to the visited nodes list. At the meanwhile, we put $B$ and $F$ into the min-heap. In this turn, the cost from root $A$ to node $B$ is 15 minutes, Figure 4(c). The next iteration picks up the node $B$ again from the min-heap $M H$. The algorithm skips the processing for $B$, as it is already in the visited node list $N L$. Accordingly, we continue the expansion of node $D$, and insert $E$ to the min-heap. After that, all the remaining nodes in the min-heap have the distance more than the time interval $\mathcal{T}$. The algorithm terminates and returns the constructed predictive tree, Figure 4(e).

Predictive Tree Expansion. As we mentioned above, the $i$ Road framework can answer predictive queries up to a maximum time boundary $\mathcal{T}$ in the future. This means, the in-hand predictive tree can not support prediction for object's trips that last longer than the specified future time $\mathcal{T}$. Therefore, to support those longer trips, we have to expand the tree to make sure that its depth under the object new location is not less than $\mathcal{T}$. In this section, we explain how to expand the predictive tree according to an object's movements.

Main idea. The main idea to expand a predictive tree is to locate the original predictive tree based on the start location of the object. After that, we take the new location of the object and make it as the new root for the just updated predictive tree. Then, the new destinations of the moving objects are the nodes located within the sub-tree underneath the new root. At the meanwhile, all the other nodes will be discarded for the further predication and processing. One important thing we have to take into consideration while we are inserting new nodes to the expanded predictive tree is that, all inserted nodes have to be reached in a shortest path from the object's start node. This means, we can postpone the deletion of the data structures used to build the original predictive tree until its corresponding object finishes its trip. This decision is to avoid reconstructing the predictive tree from scratch, instead, the expansion process acts as one more iteration in the construction algorithm.

Example. Figure 4(f) gives an example for updating a predictive tree according to the object movement. Figure 4(e) gives the original predictive tree for the object $o$ when it starts the trip at node $A$. In this example, the object moves from the starting location $A$ to $C$. We get the current location of the object and locate it to the new node $C$ in the original predictive tree. After that, the system returns $C$ directly to the moving object as the root for its expanded predictive tree. Then, only the children of the new root, i.e., node $F$, will be considered in the further processing.

\section{B. Predictive Tree Maintenance}

In this section, we explain how to maintain the constructed predictive tree such that objects movements are reflected on the structure of the tree as well as the probabilities of its nodes.

Main idea. When a moving object starts a new trip, we get its start location and map it to the closest node in the road network graph. Then, we dispatch the tree construction module to construct a predictive tree with the root pointing to the object's start node. At this point, the tree construction computes the probability of each node in the in-hand predictive tree to be a destination to that object. If a node has a probability higher than the predefined threshold parameter $\mathcal{P}$, we insert the object to the list of predicted objects stored at this node. When the object travels to its next node in the tree, the predictive tree maintenance maintains the predicted results 


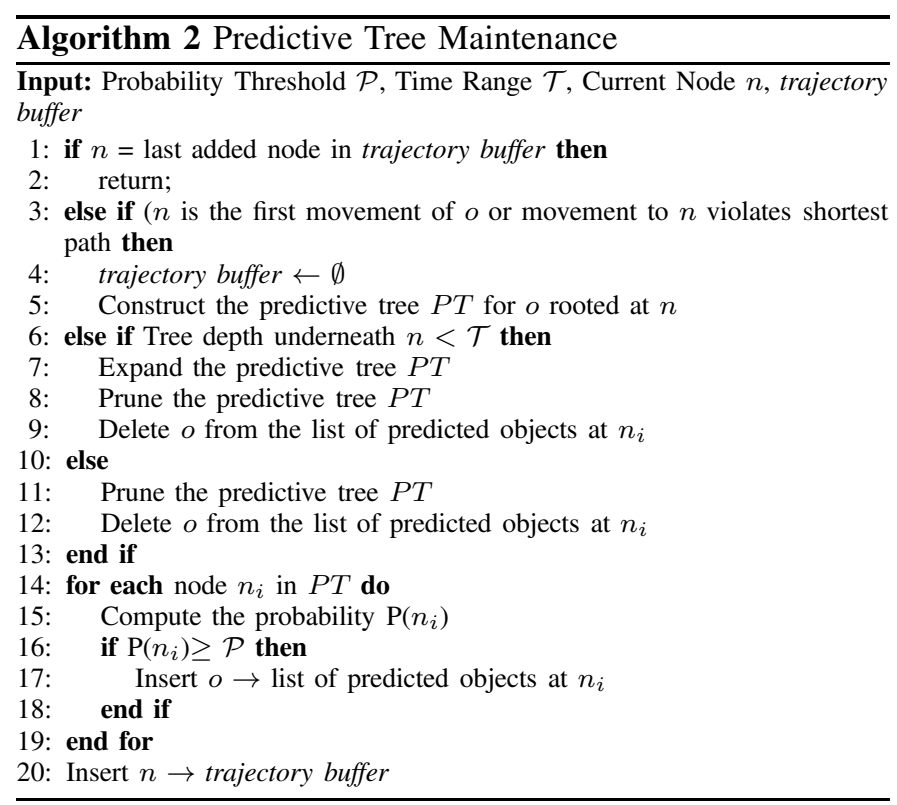

at each node in the tree as follows. (1) The predictive tree is updated by changing its root to point out to the object current node, and/or by extending the tree if its depth underneath the objects' current node is less than the time period $\mathcal{T}$. (2) All nodes other than those in the current subtree underneath the new root will be pruned. Hence, we remove all occurrences of that object along with its probability from the predicted results at those pruned nodes. (3) The list of predicted objects at the current subtree will be updated by either adding the object to it, or modifying its probability in case the object is already there. In fact, the predictive tree maintenance is a core operation inside the predictive tree index structure, as it is responsible for maintaining the tree and hence updating the predicted objects at each node in the tree and reflecting that to the nodes in the original road network graph.

Algorithm. Algorithm 2 gives the pseudo code for the predictive tree maintenance algorithm. The algorithm takes the trajectory buffer for the moving object $o$, and its current node $n$, in addition to two system parameters, the probability threshold $\mathcal{P}$ and time range for maximum predictable period in the future $\mathcal{T}$. Initially, we check if the object is still around its original node, then, the algorithm does nothing, (Line 2 in Algorithm 2). Otherwise, the algorithm preforms one or more of the following possible steps.

(1) Construct New Predictive Tree. The algorithm examines if the object starts a new trip by checking for two cases; (a) it has not passed by any previous node, or (b) its recent movement violates shortest path routing which internally means the object moves to a node that is not a child of its previous node within its predictive tree. For any of these cases, the algorithm constructs a new predictive tree $P T$ for the object $o$ with a root points to the object's current node $n$, ( Lines 4, and 5, in Algorithm 2).

(2) Maintain Existing Predictive Tree. If the object $o$ takes one more step toward its destination through a node in its original predictive tree $P T$, we need to make sure that the depth of the subtree underneath its current node $n$ is greater than or equal to the maximum prediction time $\mathcal{T}$. If this is the case, we prune the in-hand tree by cutting off all nodes not in the subtree underneath the new root $n$, Line 11 in Algorithm 2. Pruned nodes are no longer possible destinations to the object $o$ as they can not be reached in a shortest path from the object's current location. If it is not the case, the algorithm dispatches the predictive tree expansion submodule, explained earlier in this section, to extend the original tree $P T$ such that the time distance from the current node $n$ to the end of $P T$ is at least equal to $\mathcal{T}$. Then we prune the updated tree, (Lines 7 , and 8 in Algorithm 2).

(3) Assign The Probability. After having the modified predictive tree $P T$ for the object $o$, we compute the probability for each node $n_{i}$ in $P T$ being a possible destination to $o$, Line 15 in Algorithm 2. This is done according to the probability assignment model, Section II-C.

(4) Maintain The Predicted Results. The aim of this step is to make sure that the list of predicted objects stored with each node in the road network get updated according to the effect of the object's movements. This is done by performing the following two actions. (First) Within the pruning process we have to delete any occurrence for the object $o$ from the list of predicted objects at the excluded nodes, (Lines 9, and 12 in Algorithm 2). (Second) After computing the probability of each node in the current $P T$, we check if this probability is above the given probability threshold $\mathcal{P}$, we insert a new record to the list of predicted results at the node $n_{i}$, in case that $o$ is not in this list. Otherwise, we just modify its corresponding probability value. This record consists of identifier for the object $o$, its probability $\mathrm{P}\left(n_{i}\right)$ to show up at $n_{i}$, the travel time from the tree root to the node $n_{i}$, (Lines from 15 to 18 in Algorithm 2). This list of predicted results will be used later by the query processor module to evaluate users' queries. Finally, the algorithm updates the trajectory buffer to accommodate the current node $n$, Line 20 in Algorithm 2.

Example. Figure 5 illustrates how the tree maintenance algorithm works. Given the road network graph shown in Figure 5(a), and assuming that the object $o$ has started its trip at node $\mathrm{A}$, we construct its predictive tree rooted at node $\mathrm{A}$ and its depth is 20 minutes, Figure 5(b). When the object $o$ moves to the next node $\mathrm{B}$ in its trip, then the algorithm preforms some consequent actions, Figure 5(c). First, it assures that the depth of the tree under the object current node $\mathrm{B}$ is at least equal to the maximum prediction time $\mathcal{T}$, which is valid in this step. Second, it updates the list of predicted objects in all nodes in the original tree rooted at node $\mathrm{A}$. This is done by modifying the probability at nodes B, E, F, and G, and deleting the occurrence of $o$ at nodes A, C, D, H, and $\mathrm{M}$ as they are no longer possible destinations to $o$. Third, we prune the tree by cutting off all nodes that are not possible destination to $o$ via a shortest path from start node A. So, we exclude the nodes $\mathrm{A}, \mathrm{C}, \mathrm{D}, \mathrm{H}$, and $\mathrm{M}$. This step reduces the CPU cost required later to compute and update the probability at each reachable node. As the object $o$ travels to the next node F, we 


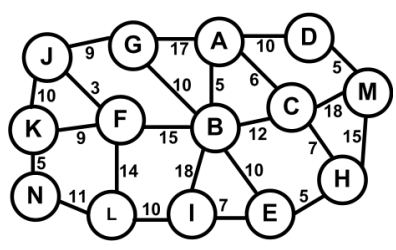

(a) Given Road Network

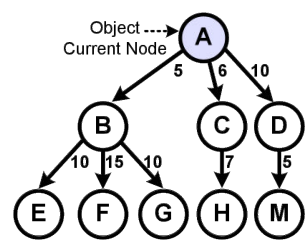

(b) Trip Start

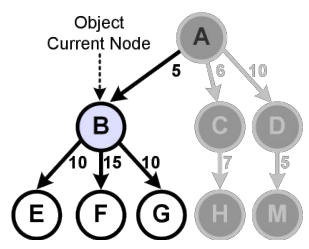

(c) Tree Pruning

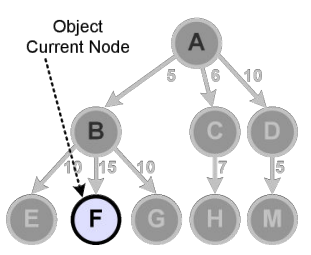

(d) No More Children

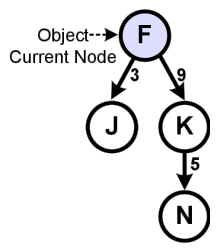

(e) Extended tree

Fig. 5. Demonstration Example For An Object Trip And Predictive Tree Maintenance

find that no further children of $\mathrm{F}$ in the current predictive tree, Figure 5(d). Yet, we need to check if it is the end of a trip, in case that the tree can not be extended. Here, it is not the case, so we extend the subtree under F, compute the probability of the nodes in this subtree, modify the list predicted objects at all nodes under node $\mathrm{B}$, and finally prune the nodes under $\mathrm{B}$ except the subtree of F, Figure 5(e).

\section{Querying The Predictive Tree}

The main idea of processing predictive queries in iRoad system is to have generic results prepared in advance and held in memory. So for coming queries, the query processor module fetches those results, adapts them according to the type of received query and responds to users in a very fast response time. Since the computation of predicted answer is already accomplished by the predictive tree construction algorithm and maintained by the predictive tree maintenance algorithm, thus, the of task of query processor module becomes straight froward. What we need to do here is, First, we find out the node of interest for which the query is asking about its predictable objects. Then, we retrieve the list of the predicted objects saved with this node. Then we shape the answer to match the user's query type. Second, we examine if the query invokes the predicted results at many nodes rather than a single node, then, we combine the answers at those nodes of interest into a single basket by taking the union of the predicted objects lists associated with them. This will get rid of redundant objects. To unify the probabilities for object predicted to appear in more than one node of interest with different probabilities, we use the maximum probability among its occurrences. Finally, according the query type,we adjust the in hand generic results.

\section{EXPERIMENTAL EVALUATION}

This section experimentally evaluates the performance of the predictive tree index within the iRoad framework.

\section{A. Experimental Setup}

In all experiments of this evaluation, we use real road network data of the Washington state, USA. For the accuracy evaluation, we use real data sets for cars trajectories around the area of Seattle [1], [14]. For the scalability and efficiency experiments, we use the Minnesota traffic generator [19] to generate large sets of synthetic moving objects on the Washington real map. The number of objects varies from $10 \mathrm{~K}$ to $50 \mathrm{~K}$ objects per set. To test the introduced approaches against different workloads, we build a query workload generator. The number of generated predictive queries varies from $10 \mathrm{~K}$ to $50 \mathrm{~K}$ queries per workload. In case of test scalability with the region sizes covered by the query, theses sizes range from 0.01 to 0.08 of the total size of the underlying road network graph. The future times $\mathcal{T}$ for the generated queries vary from 5 to 25 minutes. The probability threshold $\mathcal{P}$ varies from 0.02 to 0.10 . Predictive point query is the default query type unless we mention a different one. In each run we use $3 \mathrm{k}$ queries for warming up before we start measuring the experimental metrics, i.e, CPU cost, memory overhead, and accuracy percentage. Also, we let the system to have a sufficient number of moving objects, i.e., read all objects' movements with time stamp $\leq 5$, before we start firing the queries. All experiments are based on an actual implementation of the predictive tree and the whole iRoad framework. All the components are implemented in C\# inside visual studio 2013 with .net framework. Our implementation source code for the predictive tree, and sample datasets are accessible through [8]. All evaluations are conducted on a PC with Intel Xeon E51607 v2 processor and 32GB RAM, and running Windows 7.

\section{B. Accuracy Evaluation}

In this section, we compare the accuracy achieved by employing the predictive tree versus the Mobility Model [13]. We feed both of them with current objects trips and without historical trajectories. The prediction accuracy is measured by the probability each approach assigns to the actual future node of an object. We use real objects trajectories at five different cities around the Settle area in Washington, USA. In Figure 6, we examine the effect of the number of passed nodes on the accuracy of the next node prediction as an indicator for short term prediction. Clearly, the predictive tree gains benefits from the object's current trip by pruning the possible destinations to lower number, while the mobility model does not prune at all. Hence, the mobility model can be best described as a random walk model, in case of the absence of historical knowledge.

As given by Figure 6(d), the predictive tree can achieve up to $73 \%$ accuracy in predicting the next node. Overall, the average accuracy for the predictive tree is about $67 \%$ while it is less than $40 \%$ for the mobility model approach. The fall down in Figure 6(b) is caused by the high density of the road network in this area which increases the number of possible destinations, hence, decreases the prediction accuracy. In general, there are two main factors that affect the prediction 


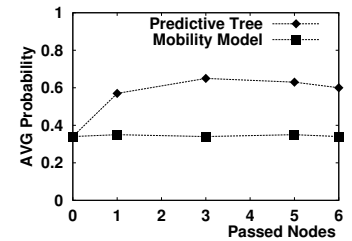

(a) Endmonds

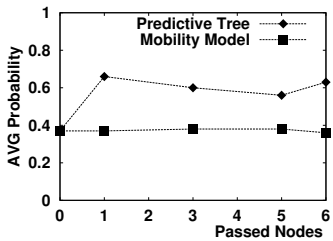

(b) Ravenna

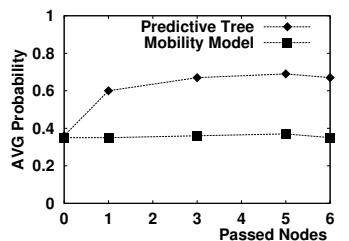

(c) Bellevue

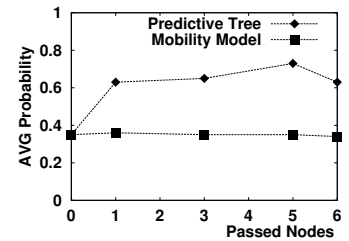

(d) Redmond

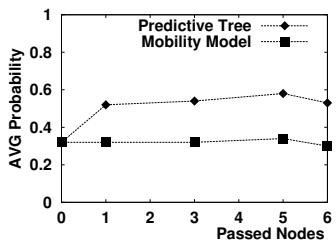

(e) Kenmore

Fig. 6. Accuracy Evaluation For Short Term Prediction

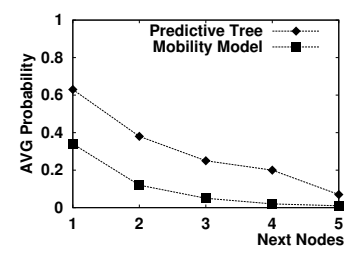

(a) Endmonds

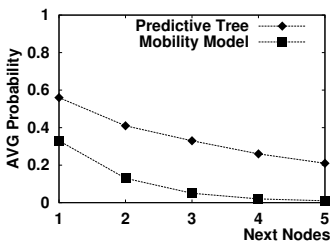

(b) Ravenna

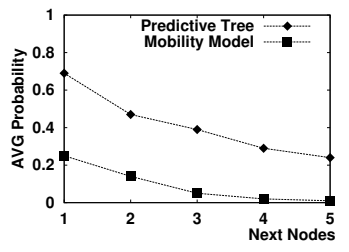

(c) Bellevue

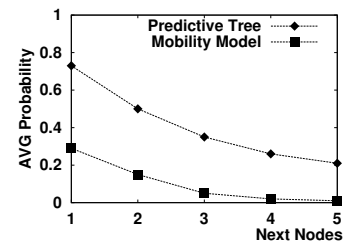

(d) Redmond

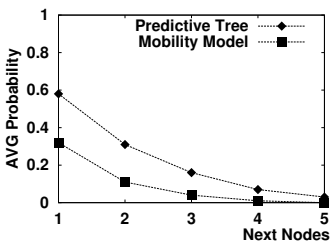

(e) Kenmore

Fig. 7. Accuracy Evaluation For Long Term Prediction

accuracy inside the predictive tree . (1) The extent to which a moving object follows the shortest paths in its travel, and (2) the average degree of a node in the underlying road network. In attempt to test the accuracy pattern of each approach in long term prediction, we let the objects pass by a sufficient number of nodes, i.e., five nodes, then we measure the probability given to each of the five nodes next to the objects' current node, Figure 7. As expected, the accuracy decreases while we look far in the future. However, the predictive tree still acts better.

\section{Scalability Evaluation}

For the scalability evaluation, we compare the two different approaches, precomputed and on-demand, of employing the predictive tree inside the iRaod framework. As discussed in Section III, the earlier is the default approach inside the iRoad framework where the predicted objects are computed and stored at each node in advance. While in the latest, we postpone all computations until a query is received. In these sets of experiments, we study the scalability of the predictive tree within the two aforementioned approaches with numbers of queries, query sizes, i.e., for predictive range queries, number of moving objects, future prediction time $\mathcal{T}$, and different levels of the probability threshold $\mathcal{P}$. For each test, we measure the average CPU time and the maximum memory footprint.

Scalability with number of queries. As depicted in Figure 8(a), the CPU cost for the on-demand approach is almost steady around 75 millisecond per query. While for the precomputed approach, it decreases from 8 millisecond per query to less than half millisecond when the number of queries increases from $20 \mathrm{~K}$ to $100 \mathrm{~K}$. This concludes that the precomputed approach significantly saves the CPU time. The reason is that once we compute and save the predicted answer at a node, it serves as many queries as needed without extra computation. However, the on-demand approach acts better w.r.t. memory overhead, Figure 9(a), as it does empty the data structures once the query is processed.

Scalability with query size. In this set of experiments, we study the scalability of each approach with large query sizes in predictive range queries. We vary the query size ratio from 0.01 to 0.08 of the the given road network graph. We found that the average CPU cost per query for the precomputed is almost steady at 3MS, while it varies from 194MS to $755 \mathrm{MS}$ for the on-demand. This means the CPU cost for the on-demand approach linearly increases when the query size increases, Figure 8(b). Intuitively, this is a rational behavior, as the on-demand performs computations for more objects with bigger sizes. Obviously, from the CPU time perspective, the precomputed approach is faster by orders of magnitude. From the memory overhead perspective, Figure 9(b), the on-demand approach is more memory friendly. However, it has some slightly increasing trend as a result of keeping the predictive trees longer time till the query is processed as a whole.

Scalability with prediction time $\mathcal{T}$. When we examine the scalability with the maximum prediction time $\mathcal{T}$ the predictive tree can support, we can notice a remarkable increase in both approaches w.r.t. the average CPU cost, Figure 8(c). However, there is a significant difference between the minimum/maximum CPU cost for each approach. The lowest CPU cost for the precomputed approach is $0.5 \mathrm{MS}$ at $\mathcal{T}=5$ minutes and 2MS for the on-demand, while the maximum is $45 \mathrm{MS}$ and 5395MS respectively at $\mathcal{T}=25$ minutes. The CPU time overheads come from the extra computations required to construct bigger predictive trees and maintain more reachable nodes. On the other hand, the memory overhead for the on-demand is almost stationary, i.e., from 19 to $22 \mathrm{MB}$. This is extremely less than the $\mathrm{min} / \mathrm{max}$ overheads for the precomputed, i.e., 219MB and 3.4GB, Figure 9(c).

Scalability with probability threshold $\mathcal{P}$. Figure $8(d)$ and Figure 9(d) illustrate the effect of increasing the probability threshold $\mathcal{P}$ from 0.02 to 0.10 on the scalability of the iRoad 


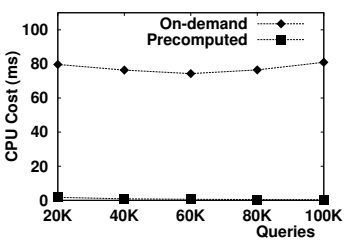

(a) Number of Queries

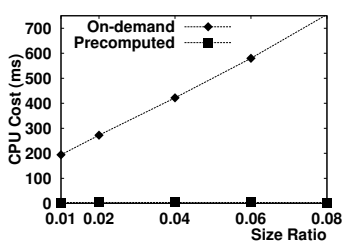

(b) Query Size

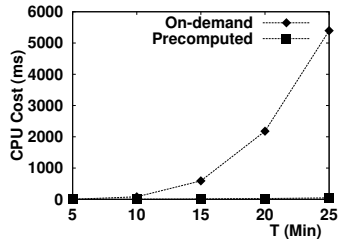

(c) Prediction Time

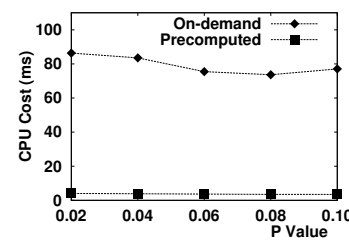

(d) Probability Threshold

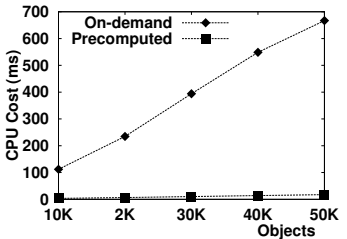

(e) Number of Objects

Fig. 8. Scalability Evaluation (CPU Time)

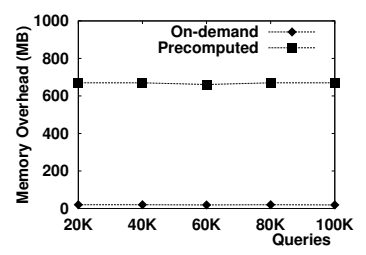

(a) Number of Queries

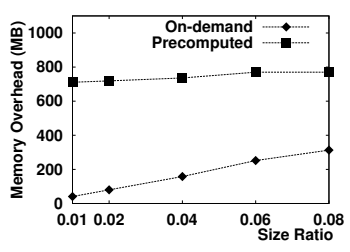

(b) Query Size

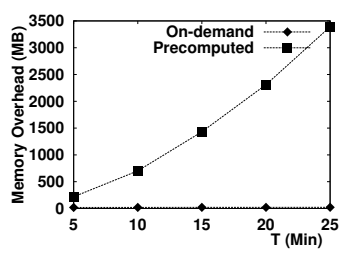

(c) Prediction Time

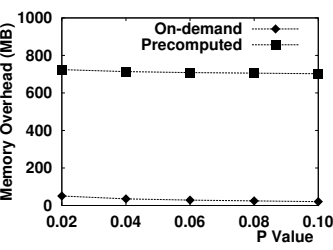

(d) Probability Threshold

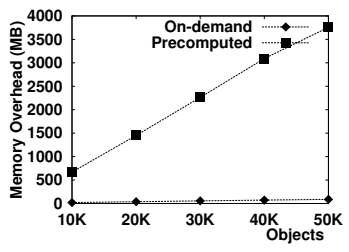

(e) Number of Objects

Fig. 9. Scalability Evaluation (Memory Overhead)

in general. For the on-demand, the average CPU cost reduces from 86 to $77 \mathrm{MS}$, and the memory overhead from 50 to 20MB. Also, for the precomputed, the average CPU cost reduces from 5 to $3 \mathrm{MS}$ and the memory overhead from 724 to $702 \mathrm{MB}$. The justification for this reduction comes from the fact that we exclude the nodes with probability less than $\mathcal{P}$.

Scalability with number of objects. The more objects to consider, the more predictive trees to build, and the extra computation and memory overhead to pay. That fact is obviously given by Figure 8(d) and Figure 9(d). The average CPU cost for the precomputed is between $4 \mathrm{MS}$ at $10 \mathrm{~K}$ objects and $18 \mathrm{MS}$ at $50 \mathrm{~K}$ objects. This is dramatically less than the one for the on-demand approach, 112 to 667MS. From the memory overhead view, the on-demand consumes 20MB more to answer the same number of queries with $10 \mathrm{~K}$ objects and $88 \mathrm{MB}$ with $50 \mathrm{~K}$ objects, while the precomputed adds up to $670 \mathrm{MB}$ with $10 \mathrm{~K}$ objects and $3 \mathrm{~GB}$ with $50 \mathrm{~K}$ objects.

\section{Efficiency Evaluation}

In this set of experiments, we evaluate the effect of the different parameters on the two main operations of the predictive tree index structure, namely tree construction and tree maintenance. We ignore querying the tree operation, i.e., query processor, since it just retrieves the answer from the query node, hence, its cost is negligible compared to the other two operations. We study those operations within the evaluation of the precomputed approach as it is the dominant w.r.t. the CPU cost. Figure 10(a) gives the impact of increasing the number of queries. We can notice the average CPU cost drops down for both operations as the number of queries goes up. As mentioned earlier, the reason is that once a predictive tree is constructed, it can participate in processing as many queries as needed without extra computation, and the same for the maintenance operation. Therefore, the average CPU time decreases while the number of queries increases. From Figure 10(b), we can conclude that both operations are significantly impacted by the maximum prediction time $\mathcal{T}$. The average CPU cost for the tree construction starts with $0.43 \mathrm{MS}$ at $\mathcal{T}=5$ minutes, and tends to $31.57 \mathrm{MS}$ at $\mathcal{T}=25$ minutes. A similar trends happens with the tree maintenance operation where it charges the CPU 0.05 and $14.03 \mathrm{MS}$ at $\mathcal{T}=5$ and 25 respectively. The impact of tuning the probability threshold $\mathcal{P}$ is provided in Figure 10(c). The major recorded influence is on the tree maintenance operation. That is because larger probability means less detailed tree, i.e., include only those nodes with probability $\geq \mathcal{P}$. We can notice that the average CPU cost for the tree maintenance drops from 0.47MS at $\mathcal{P}=$ 0.02 to $0.09 \mathrm{MS}$ at $\mathcal{P}=0.10$. Finally, Figure 10 (d) provides the consequence of large numbers of moving objects on the two fundamental operations. Normally, we construct and maintain a predictive tree for each extra moving object. Hence, the average CPU cost increases for both the tree construction and tree maintenance operations. It is also remarkable in all graphs in Figure 10 that tree construction represents the big portion of the CPU cost relative to tree maintenance operation.

\section{E. Experiments Summary}

From the accuracy evaluation set of experiments, it is obvious that the predictive tree achieves more accurate short and long term prediction compared to the most related work. From the scalability study, the predictive tree proves its ability to scale up to efficiently process heavy query workloads and handle large number of moving objects. It is also concluded that the precomputed mode is CPU friendly while the ondemand mode is memory friendly. In addition, the controllable parameters $(\mathcal{T}$ and $\mathcal{P})$ are assessed to adjust the overall system performance such that a graceful degradation takes place under periods of heavy workloads.

\section{RELATED WORK AND DISCUSSION}

In this section, we review the existing work related to predictive spatio-temporal query processing and optimization. 


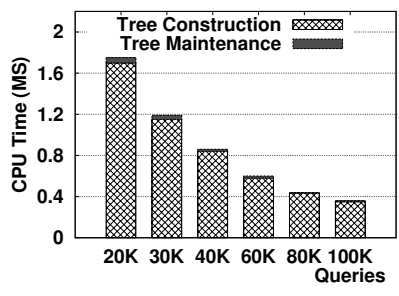

(a) Number of Queries

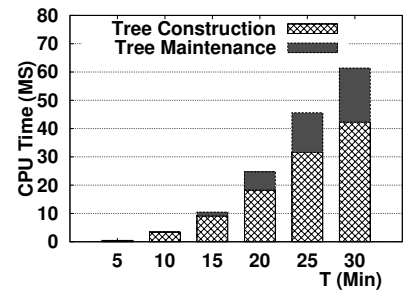

(b) Prediction Time

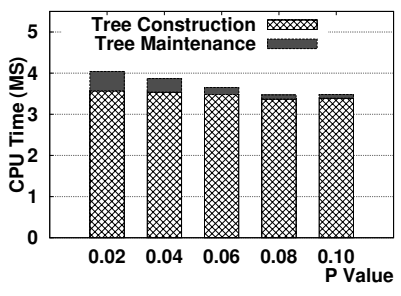

(c) Probability Threshold

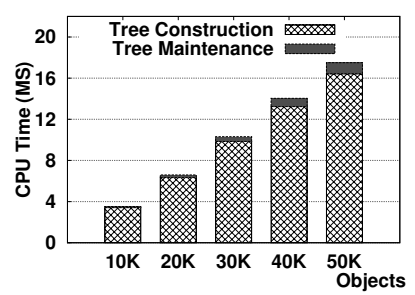

(d) Number of Objects

Fig. 10. Efficiency Evaluation Of Main Operations In The Predictive Tree

Then, we summarize the related prediction models for moving objects. This review of related work leads us to the question of how the predictive tree fits in the bigger umbrella of spatiotemporal query processing, how it is different from existing techniques and, more interestingly, how it would complement and integrate with the existing predictive query processing techniques and prediction models.

\section{A. Predictive Spatio-temporal Query Processing}

Existing algorithms for predictive query processing can be classified, according to the query type, into the following categories:

(1) Range queries, i.e., [13], [25], [30]. A predictive range query has a query region $R$ and a future time window $T$, and asks about the objects that are expected to be inside $R$ within the time window $T$. A mobility model [13] is used to predict the path of each underlying moving object that is being monitored by the system. Most existing work considers the query region to be a rectangle. However, the Transformed Minkowski Sum is used to address circular region ranges [30].

(2) K-nearest-neighbor queries, i.e., [3], [22], [30]. A predictive $K$-nearest-neighbor query has a point location $P$ and a future time window $T$, and asks about the $K$ objects expected to be closest to $P$ within the time window $T$. Two algorithms, RangeSearch and KNNSearchBF [30], are introduced to traverse a spatio-temporal index tree (the TPR/TPR ${ }^{*}$-tree) to answer range and $K \mathrm{NN}$ queries, respectively. Sometimes an expiry time interval is attached to a $k \mathrm{NN}$ query result [26], [27] to indicate the future time window during which the answer is valid.

(3) Aggregate queries, i.e., [2], [9], [24]. A predictive aggregate query has a query region $R$ and a future time window $T$, and asks about the number of objects $\mathcal{N}$ expected to be inside $R$ within the time window $T$. The approximate query processing approach in [24] employs an adaptive multidimensional histogram (AMH), a historical synopsis, and a stochastic method to provide an approximate answer for aggregate spatio-temporal queries about the future in addition to the past and the present. A generic query processing framework named Panda [9] is introduced to evaluate predictive queries for moving objects on Euclidean space.

\section{B. Prediction Models}

In fact, many prediction models have been proposed to predict the next movements of an object. One group of these prediction models are based on the Euclidean space, e.g., [9], [28], [25], [30], where objects can move freely in the two dimensional space without any constrains, i.e., without being restricted to move over edges of a road network. Furthermore, these models use Euclidean distance rather than the cost to traverse an edge within a road network graph.

Another group of prediction models that consider movements on road networks has been recently proposed, e.g., [12], [13]. The basic idea in these models is to calculate a turning probability based on applying some data mining and statistical techniques, i.e., association rules and decision trees, on the objects' historical trajectories. These models suffer from the following drawbacks: 1) The process of building a predication model requires large amount of historical trajectories, which may not be available due to privacy concerns or lack of coverage in rural areas. 2) Dispatching a data mining based model in a system that serves millions of objects places a significant overhead on computation and memory resources. Hence, responsiveness to changes in the moving objects patterns may be slow.

\section{Discussion}

To summarize, we would like to discuss how the predictive tree fits under the bigger umbrella of predictive spatiotemporal query processing and how it complements the wealth of existing techniques discussed earlier in this section. More specifically, we emphasize the following characteristics.

First, the predictive tree is a generic index structure in the sense that it is neither built for a single type of predictive queries nor for a single query processing algorithm. Rather, it provides a shared infrastructure for evaluating various types of predictive queries using various algorithms.

Second, many of the aforementioned techniques inherently depend on the object's past trajectory to accomplish the job. In the absence of such historical trajectories, the predictive tree provides a basis for these techniques to start with a predicted trajectory that is computed solely using the connectivity of the road network and, then, enhance the prediction quality as more historical data becomes available. Consequently, the aforementioned techniques are invited to utilize the predictive tree in the absence of historical data.

Third, while some of the previously mentioned systems are limited to the Euclidean space, the predictive tree is designed to handle predictive spatio-temporal queries on road networks. All computations are based on the underlying road 
map structure, i.e., using the cost of edges in the road network rather than the Euclidean distance.

Finally, the dynamic nature of the predictive tree and its incremental maintenance approach lend the proposed index naturally to the continuous query paradigm where low latency response over high rate input streams is a system priority.

\section{CONCLUSION}

In this paper, we presented the predictive tree, an index structure to handle predictive queries against moving objects on road networks. The predictive tree has been designed to offer a generic framework for a set of predictive query types, e.g., point, range and $K \mathrm{NN}$ predictive queries. The predictive tree also offers an extensibility mechanism to integrate user defined prediction models. The basic prediction model computes the moving objects expected location solely based on the connectivity of road network graph without need for objects' previous trajectories. Yet, the user can extend the prediction model to take into consideration historical trajectories of moving objects or alter the probability assignment function according to the application scenario. With the expected growth in the number of moving objects, thanks to hand-held devices and mobile phones, the predictive tree enables location-based services to scale in terms of the number of predictive queries against these moving objects. The incremental maintenance of the predictive tree guarantees minimal processing of location updates. Moreover, the behavior of the predictive tree is adjusted using a couple of tuning parameters that trade prediction accuracy for system resources, i.e., memory and CPU. The predictive tree has been implemented in the context of the iRoad system. Empirical studies proved the scalability and accuracy of the predictive tree.

\section{REFERENCES}

[1] M. Ali, J. Krumm, and A. Teredesai. ACM SIGSPATIAL GIS Cup 2012. In Proceedings of the ACM SIGSPATIAL International Conference on Advances in Geographic Information Systems, ACM SIGSPATIAL GIS, pages 597-600, California, USA, Nov. 2012.

[2] S. Ayhan, J. Pesce, P. Comitz, G. Gerberick, and S. Bliesner. Predictive Analytics with Aviation Big Data. In Proceeding of the ACM SIGSPATIAL International Workshop on Analytics for Big Geospatial Data, BigSpatial, California, USA, Nov. 2012.

[3] R. Benetis, C. S. Jensen, G. Karciauskas, and S. Saltenis. Nearest and Reverse Nearest Neighbor Queries for Moving Objects. VLDB Journal, 15(3):229-249, 2006.

[4] T. Brinkhoff. A Framework for Generating Network-Based Moving Objects. International Journal on Advances of Computer Science for Geographic Information Systems, GeoInformatica, 6(2):153-180, 2002.

[5] Y. Gu, G. Yu, N. Guo, and Y. Chen. Probabilistic Moving Range Query over RFID Spatio-temporal Data Streams. In Proceedings of the International Conference on Information and Knowledge Managemen, CIKM, pages 1413-1416, Hong Kong, China, Nov. 2009.

[6] G. Gulf. Smartphone Users Around the World Statistics and Facts. http://www.go-gulf.com/blog/smartphone/, Jan. 2012.

[7] Guttman and Antonin. R-trees: A Dynamic Index Structure for Spatial Searching. In Proceedings of the ACM International Conference on Management of Data, SIGMOD, pages 47-57, Massachusetts, USA, June 1984.

[8] A. M. Hendawi, J. Bao, and M. F. Mokbel. Predictive Tree Source Code and Sample Data. URL:http://www-users.cs.umn.edu/ hendawi/ PredictiveTree/, Aug. 2014.
[9] A. M. Hendawi and M. F. Mokbel. Panda: A Predictive Spatio-Temporal Query Processor. In Proceedings of the ACM SIGSPATIAL International Conference on Advances in Geographic Information Systems, ACM SIGSPATIAL GIS, California, USA, Nov. 2012.

[10] A. M. Hendawi and M. F. Mokbel. Predictive Spatio-Temporal Queries: A Comprehensive Survey and Future Directions. In Proceeding of the ACM SIGSPATIAL GIS International Workshop on Mobile Geographic Information Systems, MobiGIS, California, USA, Nov. 2012.

[11] H. Hu, J. Xu, and D. L. Lee. A Generic Framework for Monitoring Continuous Spatial Queries over Moving Objects. In Proceedings of the ACM International Conference on Management of Data, SIGMOD, pages 479-490, Maryland, USA, June 2005.

[12] H. Jeung, Q. Liu, H. T. Shen, and X. Zhou. A Hybrid Prediction Model for Moving Objects. In Proceedings of the International Conference on Data Engineering, ICDE, pages 70-79, Cancn, Mxico, Apr. 2008.

[13] H. Jeung, M. L. Yiu, X. Zhou, and C. S. Jensen. Path Prediction and Predictive Range Querying in Road Network Databases. VLDB Journal, 19(4):585-602, Aug. 2010.

[14] JOSM. An extensible editor for OpenStreetMap (OSM). http://josm. openstreetmap.de/wiki, Jan. 2014.

[15] H. A. Karimi and X. Liu. A Predictive Location Model for LocationBased Services. In Proceedings of the ACM Symposium on Advances in Geographic Information Systems, ACM GIS, pages 126-133, Louisiana, USA, Nov. 2003

[16] J. Krumm. Real Time Destination Prediction Based on Efficient Routes. In Proceedings of the Society of Automotive Engineers World Congress, SAE, Michigan, USA, Apr. 2006.

[17] J. Krumm. How People Use Their Vehicles: Statistics from the 2009 National Household Travel Survey. In Proceedings of the Society of Automotive Engineers World Congress, SAE, Michigan, USA, Apr. 2008.

[18] J. Krumm, R. Gruen, and D. Delling. From Destiantion Prediction To Route Prediction. Technical Report. Microsoft Research, 2011.

[19] M. F. Mokbel, L. Alarabi, J. Bao, A. Eldawy, A. Magdy, M. Sarwat, E. Waytas, and S. Yackel. MNTG: An Extensible Web-based Traffic Generator. In Proceedings of the International Symposium on Advances in Spatial and Temporal Databases, SSTD, pages 38-55, Munich, Germany, Aug. 2013.

[20] M. F. Mokbel, X. Xiong, M. A. Hammad, and W. G. Aref. Continuous Query Processing of Spatio-temporal Data Streams in PLACE. GeoInformatica, 9(4):343-365, Dec. 2005.

[21] D. Papadias, J. Zhang, and N. Mamoulis. Query Processing in Spatial Network Databases. In Proceedings of the International Conference on Very Large Data Bases, VLDB, pages 802-813, Berlin, Germany, Sept. 2003.

[22] K. Raptopoulou, A. Papadopoulos, and Y. Manolopoulos. Fast NearestNeighbor Query Processing in Moving-Object Databases. GeoInformatica, 7(2):113-137, June 2003.

[23] Stanford. Large Network Dataset Collection. http://snap.stanford.edu/ data/\#road, Jan. 2013.

[24] J. Sun, D. Papadias, Y. Tao, and B. Liu. Querying about the Past, the Present, and the Future in Spatio-Temporal. In Proceedings of the International Conference on Data Engineering, ICDE, pages 202-213, MASSACHUSETTS, USA, Mar. 2004

[25] Y. Tao, C. Faloutsos, D. Papadias, and B. L. 0002. Prediction and Indexing of Moving Objects with Unknown Motion Patterns. In Proceedings of the ACM International Conference on Management of Data, SIGMOD, pages 611-622, Paris, France, June 2004.

[26] Y. Tao and D. Papadias. Time-parameterized Queries in Spatio-temporal Databases. In Proceedings of the ACM International Conference on Management of Data, SIGMOD, pages 334-345, Wisconsin, USA, June 2002.

[27] Y. Tao and D. Papadias. Spatial queries in dynamic environments. $A C M$ Transactions on Database Systems, TODS, 28(2):101-139, 2003.

[28] Y. Tao, D. Papadias, and J. Sun. The TPR*-tree: An Optimized SpatioTemporal Access Method for Predictive Queries. In Proceedings of the International Conference on Very Large Data Bases, VLDB, pages 790801, Berlin, Germany, Sept. 2003.

[29] H. Wang, R. Zimmermann, and W.-S. Ku. Distributed Continuous Range Query Processing on Moving Objects. In Proceedings of the International Conference on Database and Expert Systems Applications, DEXA, pages 655-665, Krakow, Poland, Sept. 2006.

[30] R. Zhang, H. V. Jagadish, B. T. Dai, and K. Ramamohanarao. Optimized Algorithms for Predictive Range and KNN Queries on Moving Objects. Information Systems, 35(8):911-932, Dec. 2010. 